\title{
A CERTAIN CLASS OF APPROXIMATIONS FOR THE $q$-DIGAMMA FUNCTION
}

\author{
AHMED SALEM
}

\begin{abstract}
In this paper, we derive a class of approximations of the $q$-digamma function $\psi_{q}(x)$. The infinite family

$$
I_{a}(x ; q)=\log [x+a]_{q}+\frac{q^{x} \log q}{1-q^{x}}-\left(\frac{1}{2}-a\right) H(q-1) \log q,
$$

$a \in[0,1] ; q>0$, can be used as approximating functions for $\psi_{q}(x)$, where $[x]_{q}=\left(1-q^{x}\right) /(1-q)$ and $H(\cdot)$ is the Heaviside step function. We show that, for all $a \in[0,1], I_{a}$ is asymptotically equivalent to $\psi_{q}(x)$ for $q>0$ and is a good pointwise approximation.
\end{abstract}

1. Introduction. The $q$-analogue of the digamma function $\psi_{q}(x)$ appeared in the work of Krattenthaler and Srivastava [2] where they studied the summations for basic hypergeometric series. Some of its properties were presented and proved in their work. In [2], they proved that $\psi_{q}(x)$ tends to the digamma function $\psi(x)$ when letting $q \rightarrow 1$. Also, Salem [6] derived some properties and expansions associated with the $q$-digamma function. Some inequalities involving the $q$-digamma function have been introduced in $[\mathbf{1}, \mathbf{3}, \mathbf{7}, \mathbf{8}, \mathbf{9}, \mathbf{1 0}]$. The $q$-digamma function $\psi_{q}(x)$ is defined as the logarithmic derivative of the $q$-gamma function,

$$
\psi_{q}(x)=\frac{d}{d x}\left(\log \Gamma_{q}(x)\right)=\frac{\Gamma_{q}^{\prime}(x)}{\Gamma_{q}(x)},
$$

where $\Gamma_{q}(x)$ is the $q$-gamma function defined as

$$
\Gamma_{q}(x)=(1-q)^{1-x} \prod_{n=0}^{\infty} \frac{1-q^{n+1}}{1-q^{n+x}}, \quad 0<q<1,
$$

2010 AMS Mathematics subject classification. Primary 30E10, 33D05.

Keywords and phrases. q-Digamma function, approximations.

Received by the editors on January 17, 2013, and in revised form on November 6, 2014. 
and

$$
\Gamma_{q}(x)=(q-1)^{1-x} q^{x(x-1) / 2} \prod_{n=0}^{\infty} \frac{1-q^{-(n+1)}}{1-q^{-(n+x)}}, \quad q>1 .
$$

From (1.2), for $0<q<1$ and for all real variables $x>0$, we obtain

$$
\psi_{q}(x)=-\log (1-q)+\log q \sum_{k=0}^{\infty} \frac{q^{x k}}{1-q^{k}},
$$

and, from equation (1.3), for $q>1$ and $x>0$, we also get

$$
\psi_{q}(x)=-\log (q-1)+\log q\left[x-\frac{1}{2}-\sum_{k=0}^{\infty} \frac{q^{-x k}}{1-q^{-k}}\right] .
$$

From the previous definitions, for a positive $x$ and $q \geq 1$, we obtain

$$
\begin{aligned}
\Gamma_{q}(x) & =q^{[(x-1)(x-2)] / 2} \Gamma_{q^{-1}}(x), \\
\psi_{q}(x) & =\frac{2 x-3}{2} \log q+\psi_{q^{-1}}(x) .
\end{aligned}
$$

Muqattash and Yahdi [5] derived an infinite family of approximations for $\psi(x)$ on $\mathbb{R}_{+}$, denoted as $\left\{I_{a}, a \in[0,1]\right\}$, where

$$
I_{a}(x)=\log (x+a)-\frac{1}{x} \text {. }
$$

They proved that the functions $I_{a}$ are shown to approximate locally and asymptotically independently of $a \in[0,1]$ with a perfect match $\psi(x)=I_{a}(x)$ for a certain $a$ whenever $x$ is fixed. Also, they found local and global bounding error functions and introduced new inequalities for the digamma function.

For any real numbers $a \in[0,1]$ and $q>0$, suppose that $I_{a}(x ; q)$ is the function defined for all real positive $x$ by

$$
I_{a}(x ; q)=\log [x+a]_{q}+\frac{q^{x} \log q}{1-q^{x}}+\left(\frac{1}{2}-a\right) H(q-1) \log q,
$$

where $[x]_{q}=\left(1-q^{x}\right) /(1-q)$ is the so-called basic number and $H(\cdot)$ is the Heaviside step function.

The main goal of this paper is to derive a class of approximations of the $q$-digamma function and, as a consequence, new inequalities for the $q$-digamma function. The infinite family $I_{a}(x ; q): a \in[0,1]$ can 
be used as approximating functions for $\psi_{q}(x)$. We show that, for all $a \in[0,1], I_{a}$ is asymptotically equivalent to $\psi_{q}(x)$ for $q>0$ and is a good pointwise approximation.

2. Useful lemmas. We devote this section to establishing some preliminary facts and results needed in the proofs of the main results.

Lemma 2.1. For all $x, q \in \mathbb{R}_{+}$, we have

$$
\begin{aligned}
\log [x]_{q}+\frac{1}{2} H(q-1) \log q & \leq \psi_{q}(x+1) \\
& \leq \log [x+1]_{q}-\frac{1}{2} H(q-1) \log q .
\end{aligned}
$$

Proof. Suppose that the function

$$
f_{\alpha}(x ; q)=\psi_{q}(x+1)-\log [x+\alpha]_{q}, \quad 0<q<1, \alpha=0,1 .
$$

From equation (1.4) and the Taylor series of logarithm functions, the function $f_{\alpha}(x ; q)$ can be rewritten as

$$
f_{\alpha}(x ; q)=\sum_{k=1}^{\infty} \frac{q^{x k}}{k\left(1-q^{k}\right)} g_{\alpha}(y), \quad y=q^{k},
$$

where $g_{\alpha}(y)=y \log y+y^{\alpha}(1-y)$ can be represented as

$$
\begin{aligned}
& g_{0}(y)=y \sum_{n=2}^{\infty} \frac{\log ^{n}(1 / y)}{n !}>0 \\
& g_{1}(y)=-y^{2} \sum_{n=2}^{\infty} \frac{\log ^{n}(1 / y)}{n !}(n-1)<0 .
\end{aligned}
$$

Thus, the functions $f_{0}(x ; q)>0$ and $f_{1}(x ; q)<0$ for all $x>0$. Therefore,

$$
\log [x]_{q} \leq \psi_{q}(x+1) \leq \log [x+1]_{q}, \quad 0<q<1 .
$$

Now, let $q \geq 1$. Then equation (1.7) and the identity $[x]_{q^{-1}}=q^{x-1}[x]_{q}$ 
can be exploited to obtain

$$
\begin{aligned}
f_{\alpha}(x ; q)= & \psi_{q^{-1}}(x+1)+\frac{2 x-1}{2} \log q \\
& -\log [x+\alpha]_{q}-(x+\alpha-1) \log q \\
= & f_{\alpha}\left(x ; q^{-1}\right)+\left(\frac{1}{2}-\alpha\right) \log q, \quad q \geq 1 .
\end{aligned}
$$

In view of equations $(2.2),(2.3)$ and (2.4), we obtain the desired result.

Lemma 2.2. For every $x, q \in \mathbb{R}_{+}$, the $q$-digamma function $\psi_{q}(x)$ is strictly increasing on $(0, \infty)$, and there exists a unique real number $x^{*} \in(1,2)$ such that $\psi_{q}\left(x^{*}\right)=0$.

Proof. For $q>0$, Alzer and Grinshpan [1] stated that $\psi_{q}^{\prime}(x)$ is strictly completely monotonic on $(0, \infty)$. This means that $\psi_{q}^{\prime}(x)>0$, which reveals that $\psi_{q}(x)$ is strictly increasing on $(0, \infty)$.

When $0<q<1$, (1.4) and the Taylor expansion of $\log (1-q)$ gives

$$
\psi_{q}(1)=-\log (1-q)+\log q \sum_{k=1}^{\infty} \frac{q^{k}}{1-q^{k}}=\sum_{k=1}^{\infty} \frac{q^{k}\left(1-q^{k}+k \log q\right)}{k\left(1-q^{k}\right)} .
$$

It is easy to show that

$$
1-q^{k}+k \log q=-q^{k} \sum_{n=2}^{\infty} \frac{\log ^{n}\left(q^{-k}\right)}{n(n-2) !}<0, \quad k \in \mathbb{N},
$$

which leads to the conclusion that $\psi_{q}(1)<0$ for $0<q<1$. When $q>1$, equation $(1.7)$ gives $\psi_{q}(1)=-(1 / 2) \log q+\psi_{q^{-1}}(1)<0$. This leads to the conclusion that $\psi_{q}(1)<0$ for all $q>0$.

Similarly, we can deduce that $\psi_{q}(2)>0$ for all $q>0$. In light of this proof we conclude that there exists a unique real number $x^{*} \in(1,2)$ such that $\psi_{q}\left(x^{*}\right)=0$ for all $q>0$.

Lemma 2.3. For each real number $x, q \in \mathbb{R}_{+}$. Then, for any fixed $a \in[0,1]$, the function $x \mapsto I_{a}(x ; q)$ is positive and strictly increasing on $[2, \infty)$, and, whenever $x$ is fixed in $[2, \infty)$, the function $a \mapsto I_{a}(x ; q)$ is positive and strictly increasing on $[0,1]$. 
Proof. Differentiating equation (1.9) with respect to $x$ yields

$$
\frac{\partial}{\partial x} I_{a}(x ; q)=\frac{-q^{x+a} \log q}{1-q^{x+a}}+\frac{q^{x} \log ^{2} q}{\left(1-q^{x}\right)^{2}}>0, \quad q>0,
$$

which yields that the function $x \mapsto I_{a}(x ; q)$ is strictly increasing on $[2, \infty)$.

Differentiating equation (1.9) with respect to $a$ yields

$$
\frac{\partial}{\partial a} I_{a}(x ; q)=\frac{-q^{x+a} \log q}{1-q^{x+a}}>0, \quad 0<q<1,
$$

and

$$
\frac{\partial}{\partial a} I_{a}(x ; q)=\frac{-q^{x+a} \log q}{1-q^{x+a}}-\log q=\frac{-\log q}{1-q^{x+a}}>0, \quad q \geq 1,
$$

which yield that the function $a \mapsto I_{a}(x ; q)$ is strictly increasing on $[0,1]$. Therefore, the minimum value of $I_{a}(x ; q)$ can be computed for $0<q<1$ as

$$
\begin{aligned}
I_{0}(2 ; q) & =\log \left(1-q^{2}\right)-\log (1-q)+\frac{q^{2} \log q}{1-q^{2}} \\
& =\sum_{k=1}^{\infty} \frac{q^{k}\left(1-q^{k}+k q^{k} \log q\right)}{k} .
\end{aligned}
$$

A short calculation gives

$$
1-q^{k}+k q^{k} \log q=\sum_{n=2}^{\infty} \frac{\log ^{n}\left(q^{-k}\right)}{n !}>0,
$$

which reveals that $I_{0}(2 ; q)>0$ for $0<q<1$. When $q \geq 1$, we have

$$
I_{0}(2 ; q)=\log (1+q)+\frac{q^{2} \log q}{1-q^{2}}+\frac{1}{2} \log q .
$$

Differentiation yields $(d / d q) I_{0}(2 ; q)=g(q) /\left(2\left(1-q^{2}\right)^{2}\right)$, where

$$
g(q)=4 q^{2} \log q+\left(1-q^{2}\right)\left(1+2 q-q^{2}\right) .
$$

Again, differentiation gives

$$
g^{\prime}(q)=2-6 q^{2}+4 q^{3}+8 q \log q=2(2 q+1)(q-1)^{2}+8 q \log q>0,
$$


which concludes that $g(q)$ is increasing on $[1, \infty)$ and since $g(1)=0$, then $g(q) \geq 0$ for all $q \geq 1$. Therefore, the function $I_{0}(2 ; q)$ is increasing on $[1, \infty)$. Since $\lim _{q \rightarrow 1} I_{0}(2 ; q)=\log 2-(1 / 2)>0$, then $I_{0}(2 ; q)>0$ for all $q \geq 1$. This completes the proof.

Lemma 2.4. Suppose $q \in \mathbb{R}_{+}$. Then we have

$$
\lim _{x \rightarrow \infty} \frac{I_{0}(x ; q)}{I_{1}(x ; q)}=1 .
$$

Proof. When $0<q<1$, equation (1.9) gives

$$
\begin{aligned}
\lim _{x \rightarrow \infty} \frac{I_{0}(x ; q)}{I_{1}(x ; q)} & =\lim _{x \rightarrow \infty} \frac{\log [x]_{q}+\left(q^{x} \log q\right) /\left(1-q^{x}\right)}{\log [x+1]_{q}+\left(q^{x} \log q\right) /\left(1-q^{x}\right)} \\
& =\lim _{x \rightarrow \infty} \frac{\log \left(1-q^{x}\right)-\log (1-q)+\left(q^{x} \log q\right) /\left(1-q^{x}\right)}{\log \left(1-q^{x+1}\right)-\log (1-q)+\left(q^{x} \log q\right) /\left(1-q^{x}\right.} \\
& =\frac{-\log (1-q)}{-\log (1-q)}=1 .
\end{aligned}
$$

When $q \geq 1$, we obtain

$$
\begin{aligned}
\lim _{x \rightarrow \infty} \frac{I_{0}(x ; q)}{I_{1}(x ; q)} & =\lim _{x \rightarrow \infty} \frac{\log [x]_{q}+\left(q^{x} \log q\right) /\left(1-q^{x}\right)+1 / 2 \log q}{\log [x+1]_{q}+\left(q^{x} \log q\right) /\left(1-q^{x}\right)-1 / 2 \log q} \\
& =\lim _{x \rightarrow \infty} \frac{\log \left(q^{x}-1\right)-\log (q-1)+\left(q^{x} \log q\right) /\left(1-q^{x}\right)+1 / 2 \log q}{\log \left(q^{x+1}-1\right)-\log (q-1)+\left(q^{x} \log q\right) /\left(1-q^{x}\right)-1 / 2 \log q} \\
& =\lim _{x \rightarrow \infty} \frac{x \log q+\log \left(1-q^{-x}\right)-\log (q-1)+(\log q) /\left(q^{-x}-1\right)+1 / 2 \log q}{(x+1) \log q+\log \left(1-q^{-x-1}\right)-\log (q-1)+(\log q) /\left(q^{-x}-1\right)-1 / 2 \log q} .
\end{aligned}
$$

Using L'Hopital's rule yields

$$
\begin{aligned}
\lim _{x \rightarrow \infty} \frac{I_{0}(x ; q)}{I_{1}(x ; q)} & =\lim _{x \rightarrow \infty} \frac{\log q+\left(q^{-x}\right) /\left(1-q^{-x}\right)+\left(q^{-x} \log ^{2} q\right) /\left(q^{-x}-1\right)^{2}}{\log q+\left(q^{-x-1}\right) /\left(1-q^{-x-1}\right)+\left(q^{-x} \log ^{2} q\right) /\left(q^{-x}-1\right)^{2}} \\
& =\frac{\log q}{\log q}=1 .
\end{aligned}
$$

Lemma 2.5. For all $q>0$, the function,

$$
F_{q}(x)=\log [x]_{q}-\log [x+1 / 2]_{q}-\frac{1}{2} \frac{q^{x} \log q}{1-q^{x}},
$$

is strictly positive for all $x>0$. 
Proof. When $0<q<1$, the series expansion of the logarithm function and binomial theorem give

$$
F_{q}(x)=\frac{1}{2} \sum_{k=1}^{\infty} \frac{q^{x k}}{k} g(y), \quad y=q^{k},
$$

where $g(y)=2 \sqrt{y}-\log y-2$, which can be read as

$$
g(y)=2 \sqrt{y} \sum_{n=2}^{\infty} \frac{\log ^{n}(1 / y)}{n !}\left(\frac{1}{2}\right)^{n}(n-1)>0 .
$$

Hence, $F_{q}(x)>0$ for $0<q<1$ and $x>0$. When $q \geq 1$, it is not difficult to see that $F_{q}(x)=F_{q^{-1}}(x)$ which concludes that $F_{q}(x)>0$ for all $q>0$ and $x>0$.

Lemma 2.6. For all $q>0$, the function

$$
G_{q}(x)=\frac{1}{2} \log [x+1 / 2]_{q}+\frac{1}{2} \log [x]_{q}+\frac{3}{4} \frac{q^{x} \log q}{1-q^{x}}-\psi_{q}(x),
$$

is strictly positive for all $x>0$.

Proof. The function $G_{q}(x)$ can be represented by using (1.4) as

$$
G_{q}(x)=-\frac{1}{4} \sum_{k=1}^{\infty} \frac{q^{x k}}{k\left(1-q^{k}\right)} h(y), \quad y=q^{k} ; 0<q<1,
$$

where

$$
h(y)=\log y+3 y \log y+2 \sqrt{y}(1-y)+2(1-y),
$$

which can be read as

$$
\begin{aligned}
h(y)=-y \sqrt{y} \sum_{n=3}^{\infty} & \frac{\log ^{n}(1 / y)}{n !} \\
& \left\{\left(\frac{3}{2}\right)^{n-1}(n-3)+\left(\frac{1}{2}\right)^{n-1}(3 n+1)-2\right\}<0,
\end{aligned}
$$

which reveals that $G_{q}(x)>0$ for $0<q<1$ and $x>0$. When $q \geq 1$, (1.7) gives $G_{q}(x)=G_{q^{-1}}(x)$, and so $G_{q}(x)>0$ for $q>0$ and $x>0$. This ends the proof. 
3. The main results. In this section, we are seeking to derive an infinite family of approximations of the $q$-digamma function and, as a consequence, new inequalities for the $q$-digamma function. The infinite family $I_{a}(x ; q): a \in[0,1]$ can be used as approximating functions for $\psi_{q}(x)$. We show that, for all $a \in[0,1], I_{a}$ is asymptotically equivalent to $\psi_{q}(x)$ for $q>0$ and is a good pointwise approximation. In order to present our proofs, we will use the lemmas proved in the previous section and the same technique used in [5].

Theorem 3.1. For every $x, q \in \mathbb{R}_{+}$, there exists at least one real number $a \in[0,1]$ such that

$$
\psi_{q}(x)=I_{a}(x ; q) .
$$

Proof. The intermediate value theorem states that, for each value between the least upper bound and greatest lower bound of the image of a continuous function, there is at least one point in its domain which the function maps to that value. It is clear that the function $a \mapsto I_{a}(x ; q)$ is a continuous function for all $a \in[0,1]$. From equation (1.9) and Lemma 2.1, along with the identity [7],

$$
\psi_{q}(x+1)=\psi_{q}(x)-\frac{q^{x} \log q}{1-q^{x}} .
$$

we have

$$
I_{0}(x ; q) \leq \psi_{q}(x) \leq I_{1}(x ; q) .
$$

According to the intermediate value theorem, we conclude that at least one real number $a \in[0,1]$ exists such that $\psi_{q}(x)=I_{a}(x ; q)$. This concludes the proof.

As in [5], we will use the notation $f \sim g$ on $\mathbb{R}_{+}$to denote that the functions $f$ and $g$ are asymptotic.

Theorem 3.2. For all $a \in[0,1]$ and $q>0$, then $\psi_{q}(x) \sim I_{a}(x ; q)$ on $\mathbb{R}_{+}$. 
Proof. Dividing the inequality $(3.3)$ by $I_{1}(x ; q)$ which is a positive for all $x \in[2, \infty)$, see Lemma 2.3 , would yield

$$
\frac{I_{0}(x ; q)}{I_{1}(x ; q)} \leq \frac{\psi_{q}(x)}{I_{1}(x ; q)} \leq 1 .
$$

Exploiting equation (2.5) gives

$$
\lim _{x \rightarrow \infty} \frac{\psi_{q}(x)}{I_{1}(x ; q)}=1,
$$

and thus,

$$
\psi_{q}(x) \sim I_{1}(x ; q) \quad \text { on } \mathbb{R}_{+} .
$$

Similarly, we can deduce that

$$
\lim _{x \rightarrow \infty} \frac{\psi_{q}(x)}{I_{0}(x ; q)}=1,
$$

and thus,

$$
\psi_{q}(x) \sim I_{0}(x ; q) \quad \text { on } \mathbb{R}_{+} .
$$

In view of Lemmas 2.2 and 2.3 , we see, for all $x \geq 2, q>0$ and for all $a \in[0,1]$, that

$$
0<I_{0}(x ; q) \leq I_{a}(x ; q) \leq I_{1}(x ; q),
$$

and $\psi_{q}(x)>0$ for all $x \geq 2$ and $q>0$. Therefore,

$$
\frac{\psi_{q}(x)}{I_{1}(x ; q)} \leq \frac{\psi_{q}(x)}{I_{a}(x ; q)} \leq \frac{\psi_{q}(x)}{I_{0}(x ; q)} .
$$

In view of equations (3.4), (3.5) and (3.7), we conclude that

$$
\lim _{x \rightarrow \infty} \frac{\psi_{q}(x)}{I_{a}(x ; q)}=1,
$$

and thus,

$$
\psi_{q}(x) \sim I_{a}(x ; q) \quad \text { on } \mathbb{R}_{+} .
$$

This ends the proof.

We are now interested in studying the error of the approximation. 
Definition 3.3. Suppose that $x \in[2, \infty), q \in \mathbb{R}_{+}$and $a \in[0,1]$. We define

$$
E_{a}(x ; q)=\psi_{q}(x)-I_{a}(x ; q)
$$

as the error of the approximation $\psi_{q}(x) \approx I_{a}(x ; q)$.

Theorem 3.4. For any $a \in[0,1]$ and $q \in \mathbb{R}_{+}$, the error $E_{a}(x ; q)$ approaches zero as $x \rightarrow \infty$, and therefore $\psi_{q}(x) \approx I_{a}(x ; q)$ for relatively large $x$.

Proof. In view of equations (3.3) and (3.6), we deduce for $a \in[0,1]$, $q \in \mathbb{R}_{+}$and $x \in[2, \infty)$ that

$$
\left|\psi_{q}(x)-I_{a}(x ; q)\right| \leq I_{1}(x ; q)-I_{0}(x ; q),
$$

or equivalently,

$$
0 \leq\left|E_{a}(x ; q)\right| \leq \log \left(\frac{1-q^{x+1}}{1-q^{x}}\right)-H(q-1) \log q .
$$

Taking the limits as $x \rightarrow \infty$ when $0<q<1$ gives

$$
\lim _{x \rightarrow \infty}\left|E_{a}(x ; q)\right|=\lim _{x \rightarrow \infty} \log \left(\frac{1-q^{x+1}}{1-q^{x}}\right)=0,
$$

and when $q \geq 1$, gives

$$
\begin{aligned}
\lim _{x \rightarrow \infty}\left|E_{a}(x ; q)\right| & =\lim _{x \rightarrow \infty} \log \left(\frac{q^{x+1}-1}{q^{x}-1}\right)-\log q \\
& =\lim _{x \rightarrow \infty} \log \left(\frac{1-q^{-x-1}}{1-q^{-x}}\right)=0 .
\end{aligned}
$$

By virtue of equations (3.10) and (3.11) we conclude, for $q>0$, that

$$
\lim _{x \rightarrow \infty} E_{a}(x ; q)=0 .
$$

Theorem 3.5. For any $x \in[2, \infty), a \in[0,1]$ and $q \in \mathbb{R}_{+}$, we have

(i) the errors $E_{a}(x ; q)$ are uniformly bounded between $-\log (3 / 2)$ and $\log (3 / 2)$. 
(ii)

$$
\begin{aligned}
\psi_{q}(x)= & \log [x+a]_{q}+\frac{q^{x} \log q}{1-q^{x}}-\left(\frac{1}{2}+a\right) H(q-1) \log q \\
& +O\left(\log \left(q^{-H(q-1)}\left(1+\frac{q^{x}}{[x]_{q}}\right)\right)\right) .
\end{aligned}
$$

Proof. Define the function

$$
\alpha(x, q)=\log [x+1]_{q}-\log [x]_{q}-H(q-1) \log q .
$$

Differentiation gives, for all $q>0$,

$$
\frac{\partial}{\partial x} \alpha(x, q)=-\frac{q^{x+1} \log q}{1-q^{x+1}}+\frac{q^{x} \log q}{1-q^{x}}=\frac{q^{x}(1-q) \log q}{\left(1-q^{x+1}\right)\left(1-q^{x}\right)}<0,
$$

which reveals that the function $\alpha(x, q)$ is decreasing on $[2, \infty)$ for all $q>0$ with a maximum of

$$
\alpha(2, q)=\log \left(1+q+q^{2}\right)-\log (1+q)-H(q-1) \log q .
$$

When $0<q<1$, we get

$$
\frac{d}{d q} \alpha(2, q)=\frac{q^{2}+2 q}{(1+q)\left(1+q+q^{2}\right)}>0,
$$

which shows that $\alpha(2, q)$ is increasing function on $(0,1)$ onto $(0, \log (3 / 2))$. When $q>1$, we get

$$
\frac{d}{d q} \alpha(2, q)=\frac{-1-2 q}{q(1+q)\left(1+q+q^{2}\right)}<0,
$$

which reveals that $\alpha(2, q)$ is a decreasing function on $(1, \infty)$ onto $(0, \log (3 / 2))$.

In view of the previous information, we can conclude that the function $\alpha(x, q)$ is bounded between zero and $\log (3 / 2)$. Therefore, $\left|E_{q}(x ; q)\right|<\log (3 / 2)$. The second case follows immediately from equations (1.9) and (3.9).

Remark 3.6. It is worth mentioning that Moak [4] proved the following approximation for the $q$-digamma function:

$$
\psi_{q}(x)=\log [x]_{q}+\frac{1}{2} \frac{q^{x} \log q}{1-q^{x}}+O\left(\frac{q^{x} \log ^{2} q}{\left(1-q^{x}\right)^{2}}\right)
$$


holds for all $q>0$ and $x>0$ and so $\psi_{q}(x) \sim I(x ; q)$ on $\mathbb{R}_{+}$where

$$
I(x ; q)=\log [x]_{q}+\frac{1}{2} \frac{q^{x} \log q}{1-q^{x}} .
$$

In the following theorem, we will show that the approximation $I_{1 / 2}(x ; q)$ of $\psi_{q}(x)$ is better than the approximation (of Moak) $I(x ; q)$ of $\psi_{q}(x)$ for all $q>0$ and $x>0$.

Theorem 3.7. For all $x, q \in \mathbb{R}_{+}$such that $x \geq 2$, the error of approximation $I_{1 / 2}(x ; q)$ of $\psi_{q}(x)$ is less than the error of approximation $I(x ; q)$ of $\psi_{q}(x)$.

Proof. Lemma 2.5 gives that

$$
-\log [x]_{q}-1 / 2 \frac{q^{x} \log q}{1-q^{x}}<-\log [x+1 / 2]_{q}-\frac{q^{x} \log q}{1-q^{x}}
$$

which can be rewritten as

$$
\psi_{q}(x)-\log [x]_{q}-\frac{1}{2} \frac{q^{x} \log q}{1-q^{x}}<\psi_{q}(x)-\log [x+1 / 2]_{q}-\frac{q^{x} \log q}{1-q^{x}}
$$

or equivalently,

$$
\psi_{q}(x)-I(x ; q)<\psi_{q}(x)-I_{1 / 2}(x ; q) .
$$

Also, from Lemma 2.6, we have

$$
\psi_{q}(x)-\log [x+1 / 2]_{q}-\frac{q^{x} \log q}{1-q^{x}}<\log [x]_{q}+\frac{1}{2} \frac{q^{x} \log q}{1-q^{x}}-\psi_{q}(x),
$$

which is equivalent to

$$
\psi_{q}(x)-I_{1 / 2}(x ; q)<I(x ; q)-\psi_{q}(x) .
$$

Combining equations (3.13) and (3.14) yields

$$
\left|\psi_{q}(x)-I_{1 / 2}(x ; q)\right|<\left|\psi_{q}(x)-I(x ; q)\right| .
$$

Equivalently,

$$
\left|E_{1 / 2}(x ; q)\right|<\left|\psi_{q}(x)-I(x ; q)\right| \text {. }
$$


Acknowledgments. I am very grateful to the referee(s) for valuable comments and useful suggestions, which greatly helped to improve the presentation and the quality of the paper.

\section{REFERENCES}

1. H. Alzer and A.Z. Grinshpan, Inequalities for the gamma and q-gamma functions, J. Approx. Theor. 144 (2007), 67-83.

2. C. Krattenthaler and H.M. Srivastava, Summations for basic hypergeometric series involving a q-analogue of the digamma function, Comp. Math. Appl. 32 (1996), 73-91.

3. T. Mansour and A.SH. Shabani, Some inequalities for the q-digamma function, J. Inequal. Pure Appl. Math. 10 (2009), 1-8.

4. D.S. Moak, The q-analogue of Stirling's formula, Rocky Mountain J. Math. 14 (1984), 403-413.

5. I. Muqattash and M. Yahdi, Infinite family of approximations of the digamma function, Math. Comp. Mod. 43 (2006), 1329-1336.

6. A. Salem, Some properties and expansions associated with q-digamma function, Quaest. Math. 36 (2013), 67-77.

7. Complete monotonicity properties of functions involving q-gamma and q-digamma functions, Math. Inequal. Appl. 17 (2014), 801-811.

8. Two classes of bounds for the q-gamma and the q-digamma functions in terms of the q-zeta functions, Banach J. Math. Anal. 8 (2014), 109-117.

9. , An infinite class of completely monotonic functions involving the q-gamma function, J. Math. Anal. Appl. 406 (2013), 392-399.

10. digamma functions, J. Approx. Theor. 164 (2012), 971-980.

Department of Mathematics, Faculty of Science, King Abdulaziz UniverSity, JedDah 21589, SAudi Arabia

Email address: ahmedsalem74@hotmail.com 